\title{
Synchronization of Small Oscillations in Cross-Coupled Chaotic Circuits
}

\author{
Yumiko Uchitani and Yoshifumi Nishio \\ Department of Electrical and Electronic Engineering, Tokushima University \\ Email: \{uchitani, nishio\}@ee.tokushima-u.ac.jp
}

\begin{abstract}
In this study, we investigate synchronization states of small oscillations observed from simple two chaotic circuits cross-coupled by inductors by both computer simulations and circuit experiments. We confirm that there are many different synchronization states coexist.
\end{abstract}

\section{INTRODUCTION}

Synchronization phenomena in complex systems are very good models to describe various higher-dimensional nonlinear phenomena in the field of natural science. Studies on synchronization phenomena of coupled chaotic circuits are extensively carried out in various fields [1][2]. We consider that it is very important to investigate the phenomena related with chaos synchronization to realize future engineering application utilizing chaos.

In our past studies, two simple chaotic circuits cross-coupled by inductors are investigated. As a result, we could observe interesting state transition phenomena [3][4]. In particular, we noticed that small oscillations between transitions from positive region to negative region tend to be synchronized in anti-phase in spite of the synchronization of the transitions.

In this study, we investigate different synchronization states corresponding to anti-phase synchronizations of small oscillations between the transitions. We can see that the quadraturephase synchronization in [4] is one of many different synchronization states. The computer simulation results are verified by real circuit experiments and we also carry out computer simulations for Chua's circuit in order to confirm some kinds of universality of the phenomenon.

\section{CIRCUIT MODEL}

Figure 1 shows the circuit model [3]. In the circuit, two Shinriki-Mori chaotic circuits [5][6] are cross-coupled via inductors $L_{2}$.

By using the following variables and the parameters,

$$
\left\{\begin{array}{l}
x_{k}=\sqrt{\frac{L_{1}}{C_{2}}} \frac{i_{1 k}}{V}, \quad w_{k}=\sqrt{\frac{L_{1}}{C_{2}}} \frac{i_{2 k}}{V}, \\
y_{k}=\frac{v_{1 k}}{V}, \quad z_{k}=\frac{v_{2 k}}{V}, \quad t=\sqrt{L_{1} C_{2}} \tau, \\
\alpha=\frac{C_{2}}{C_{1}}, \quad \beta=\sqrt{\frac{L_{1}}{C_{2}}} G, \quad \gamma=\sqrt{\frac{L_{1}}{C_{2}}} g, \\
\delta=\frac{L_{1}}{L_{2}}, \quad " . "=\frac{d}{d \tau}
\end{array}\right.
$$

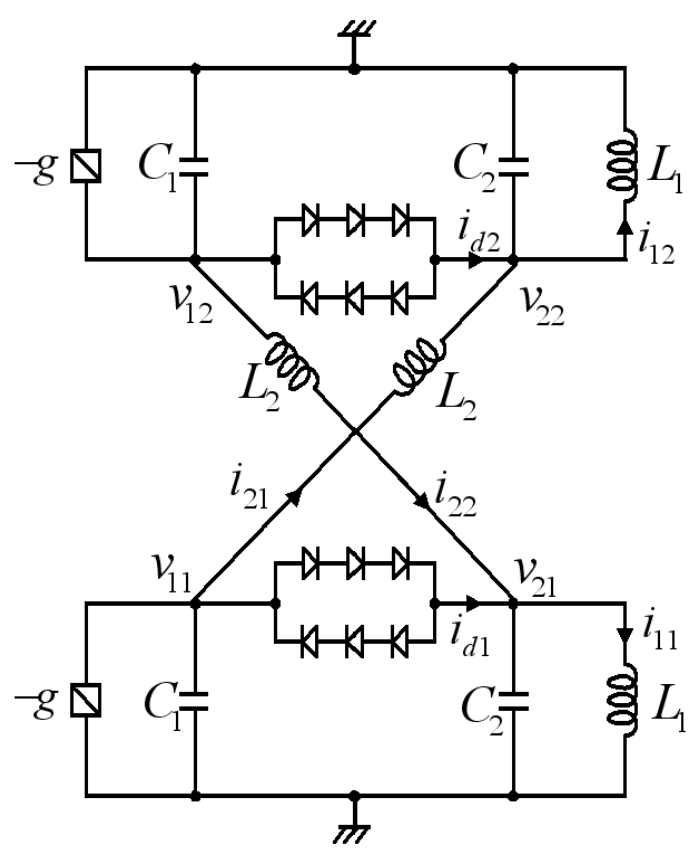

Fig. 1. Circuit model.

the normalized circuit equations are given as follows.

$$
\left\{\begin{array}{l}
\dot{x}_{1}=z_{1} \\
\dot{x}_{2}=z_{2} \\
\dot{y}_{1}=\alpha\left\{\gamma y_{1}-w_{1}-\beta f\left(y_{1}-z_{1}\right)\right\} \\
\dot{y}_{2}=\alpha\left\{\gamma y_{2}-w_{2}-\beta f\left(y_{2}-z_{2}\right)\right\} \\
\dot{z}_{1}=\beta f\left(y_{1}-z_{1}\right)+w_{2}-x_{1} \\
\dot{z}_{2}=\beta f\left(y_{2}-z_{2}\right)+w_{1}-x_{2} \\
\dot{w}_{1}=\delta\left(y_{1}-z_{2}\right) \\
\dot{w}_{2}=\delta\left(y_{2}-z_{1}\right)
\end{array}\right.
$$

where $f$ are the nonlinear functions corresponding to the $v-$ $i$ characteristics of the nonlinear resistors consisting of the diodes and are assumed to be described by the following 3segment piecewise-linear functions:

$$
f\left(y_{k}-z_{k}\right)= \begin{cases}y_{k}-z_{k}-1 & \left(y_{k}-z_{k}>1\right) \\ 0 & \left(\left|y_{k}-z_{k}\right| \leq 1\right) \\ y_{k}-z_{k}+1 & \left(y_{k}-z_{k}<-1\right)\end{cases}
$$




\section{State Transition Phenomenon}

From the circuit in Fig. 1, we could observe interesting state transition phenomenon [3][4]. A typical example of the observed phenomena is shown in Fig. 2. Figure 2(a) is computer simulated results obtained by integrating Eq. (2) with the Runge-Kutta method and Fig. 2(b) is the corresponding circuit experimental results. In this state, the two circuits exhibited chaos but almost synchronized in in-phase in the sense that the attractor was almost in the quadrant I or III on the $y_{1}$ vs $y_{2}$ (or $v_{11}$ vs $v_{21}$ ) plane. The behaviors of the circuits are very interesting because the solutions on the $y_{i} v s z_{i}$ planes seem to be attracted to the fixed points located at around $\left(y_{i}\right.$, $\left.z_{i}\right)=( \pm 1.2,0)$. However, after converging to the fixed points, the solution abruptly moves toward the other fixed point. When one circuit switches to/from the positive region from/to the negative region in this way, the other follows the transition after a few instants.

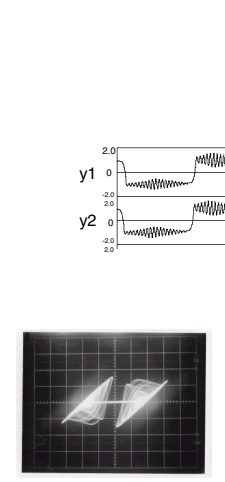

(b1)

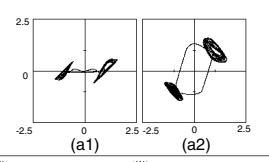

(a1) $\quad$ (a2)
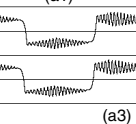

(a3)

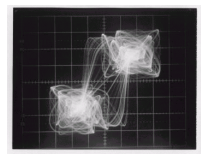

(b2) (b3)

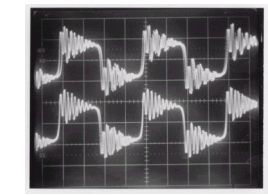

Fig. 2. State transition phenomenon around in-phase synchronization. (a) Computer calculated results. $\alpha=2.5, \beta=4.0, \gamma=0.1$, and $\delta=0.0014$. (b) Circuit experimental results. $L_{1}=9.93 \mathrm{mH}, L_{2}=$ $800 \mathrm{mH}, C_{1}=32.8 \mathrm{nF}$, and $C_{2}=49.5 \mathrm{nF}$, and $g=683 \mathrm{mS}$. (a1) $y_{1}$ vs $z_{1}$. (a2) $y_{1}$ vs $y_{2}$. (a3) Time waveform. (b1) $v_{11}$ vs $v_{12}$. (b2) $v_{11}$ vs $v_{21}$. (b3) Time waveform $v_{11}$ and $v_{21}$.

By changing initial conditions, similar transition phenomena can be observed around anti-phase synchronization and quadrature-phase synchronization as shown in Fig. 3.

Figure 4 shows the magnification of the time waveform of $y$. We can see that the switching timing of $y_{1}$ and $y_{2}$ are almost synchronized in in-phase, however, small oscillations between the transitions are synchronized in anti-phase.

\section{INVESTIGATION OF SMALL OSCILLATIONS}

In this study, we pay our attentions on the synchronization of the small oscillations between the transitions. If all the states corresponding to the anti-phase synchronizations of the small oscillations can be stable, we can observe as many different synchronization states as the number of the small oscillations between the transitions instead of just three (in-phase, antiphase, quadrature-phase) synchronizations.

Figures 5 shows some examples of different synchronization states obtained for the same parameter values. In Fig. 5(a), the

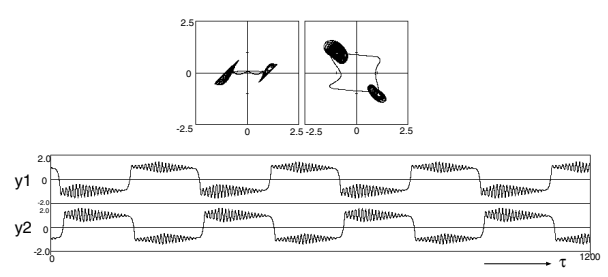

(1a)

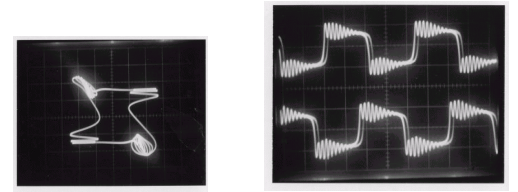

(1b)

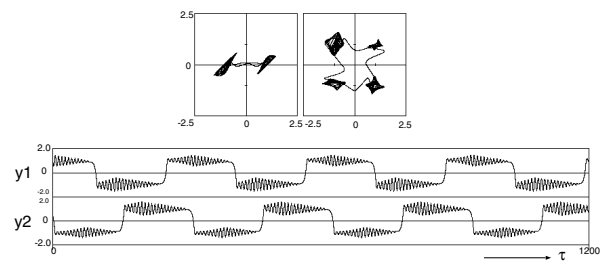

(2a)
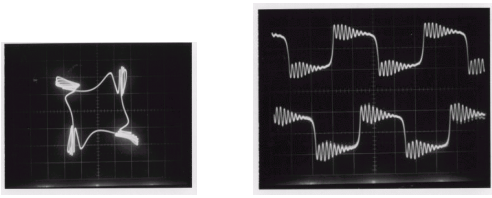

(2b)

Fig. 3. State transition phenomenon around (1) anti-phase synchronization and (2) quadrature-phase synchronization. (a) Computer calculated results. $\alpha=2.5, \beta=4.0, \gamma=0.1$, and $\delta=$ 0.0014. (b) Circuit experimental results. $L_{1}=9.93 \mathrm{mH}, L_{2}=1.2 \mathrm{H}$ $C_{1}=32.8 \mathrm{nF}, C_{2}=49.5 \mathrm{nF}$, and $g=495 \mathrm{mS}$.

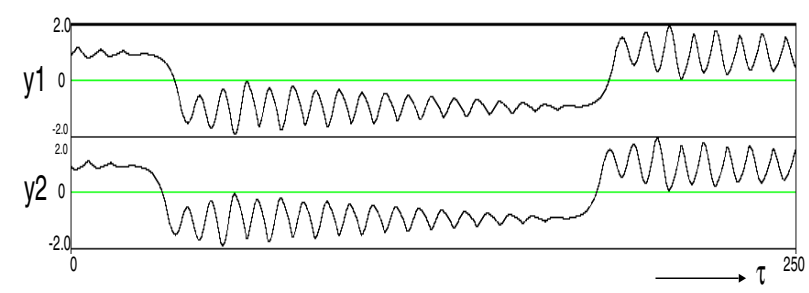

Fig. 4. Magnification of the time waveform around transition. $\alpha=$ $1.5, \beta=5.0, \gamma=0.2$, and $\delta=0.003$.

first peak of $y_{2}$ after its transition from negative to positive is synchronized to the second bottom of $y_{1}$. The first peak of $y_{2}$ of Fig. 5(b) and (c) are synchronized to the 9th and the 15th bottoms of $y_{1}$, respectively.

As we expected, we could observe many different types of the synchronization states, although we do not say that we can always observe exactly same number of the states as the number of the small oscillations between the transition.

Figure 6 shows two examples of the different synchronization states observed from real circuit experiments. 
(1)
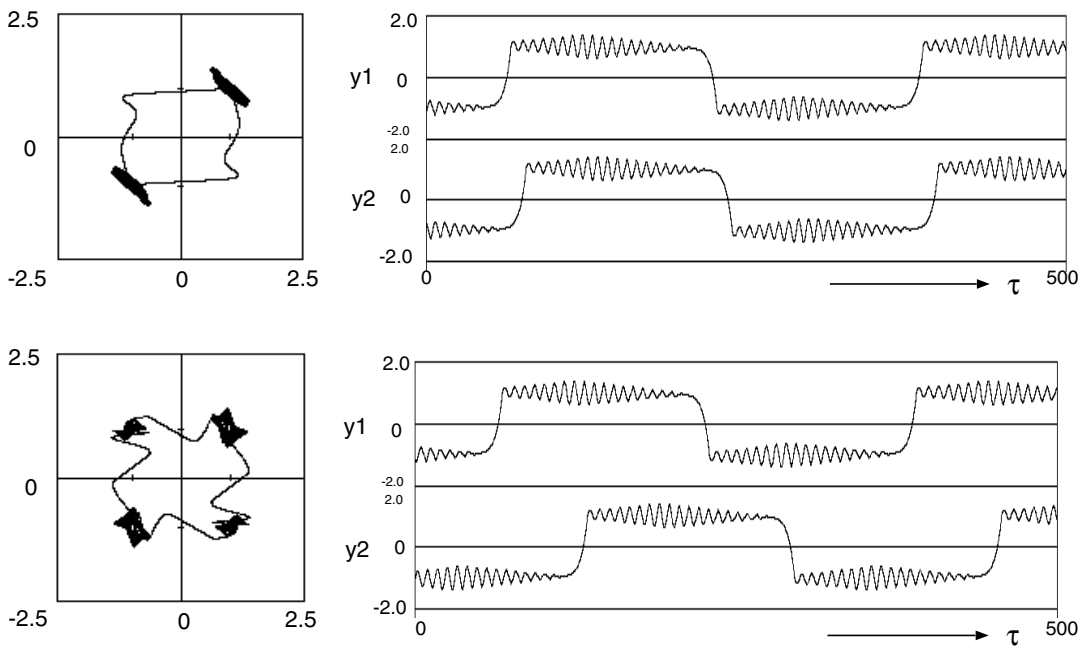

(2)

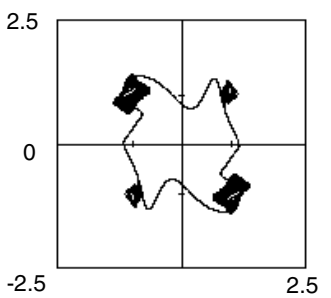

(a)

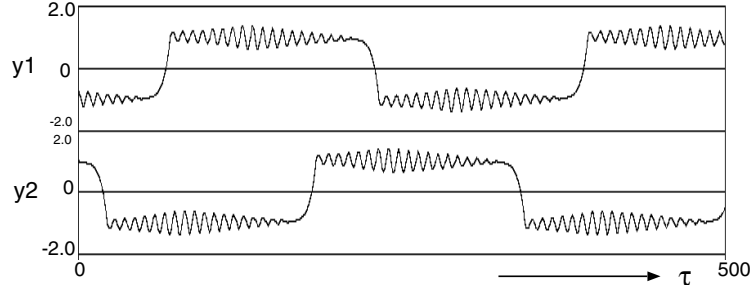

(b)

Fig. 5. Some examples of different synchronization states. (computer simulation results). $\alpha=2.0, \beta=4.0, \gamma=0.1$, and $\delta=0.0014$. (1) First peak of $y_{2}$ is synchronized to the second bottom of $y_{1}$. (2) Ninth bottom of $y_{1}$. (3) Fifteenth bottom of $y_{1}$. (a) Attractor on $y_{1} v s y_{2}$ plane. (b) Timewaveform.

\section{ChuA's CiRCuit CASE}

In order to show that the synchronization in the previous section is not special only for the chaotic circuit in Fig. 1, we carried out similar computer simulations for the well-known Chua's circuit (Figs. 7 and 8).

The circuit equations can be written after an appropriate nondimensional variables and parameters as follows:

$$
\left\{\begin{array}{l}
\dot{x}_{1}=-\beta z_{1} \\
\dot{x}_{2}=-\beta z_{2} \\
\dot{y}_{1}=\alpha\left\{-y_{1}+z_{1}-w_{1}-f\left(y_{1}\right)\right\} \\
\dot{y}_{2}=\alpha\left\{-y_{2}+z_{2}-w_{2}-f\left(y_{2}\right)\right\} \\
\dot{z}_{1}=x_{1}+y_{1}-z_{1}+w_{2} \\
\dot{z}_{2}=x_{2}+y_{2}-z_{2}+w_{1} \\
\dot{w}_{1}=\beta \delta\left(y_{1}-z_{2}\right) \\
\dot{w}_{2}=\beta \delta\left(y_{2}-z_{1}\right)
\end{array}\right.
$$

where $f$ are the nonlinear functions corresponding to the $v-i$ characteristics of the Chua diode and can be described by 3 segment piecewise-linear functions.

Figures 9 shows some examples of computer calculated results. We could observe similar synchronization phenomena from the coupled Chua's circuits, namely several different synchronization states characterized by anti-phase synchronization of the small oscillations between the transitions.

\section{CONCLUSIONS}

In this study, we have investigated different synchronization states corresponding to anti-phase synchronizations of small oscillations between the transitions observed from simple chaotic circuits cross-coupled by inductors. By computer simulations and circuit experiments, we confirmed that the circuits generated many different synchronization states characterized by the number of the small oscillations between the transitions.

\section{REFERENCES}

[1] G. Abramson,V.M. Kenkre and A.R. Bishop, "Analytic Solutions for Nonlinear Waves in Coupled Reacting Systems," Physica A, vol. 305, no. 3-4, pp. 427-436, 2002.

[2] I. Belykh, M. Hasler, M. Lauret and H. Nijmeijer, "Synchronization and Graph Topology," Int. J. Bifurcation and Chaos, vol. 15, no. 11, pp. 3423-3433, 2005.

[3] Y. Uchitani, R. Imabayashi and Y. Nishio, "State Transition Phenomenon in Cross-Coupled Chaotic Circuits," Proc. of NOLTA'07, pp. 397-400, Sep. 2007.

[4] Y. Uchitani and Y. Nishio, "Investigation of State Transition Phenomena in Cross-Coupled Chaotic Circuits," Proc. of ISCAS'08, pp. 2394-2397, May. 2008.

[5] M. Shinriki, M. Yamamoto and S. Mori, "Multimode Oscillations in a Modified van der Pol Oscillator Containing a Positive Nonlinear Conductance," Proc. of IEEE, vol. 69, pp. 394-395, 1981.

[6] N. Inaba, T. Saito and S. Mori, "Chaotic Phenomena in a Circuit with a Negative Resistance and an Ideal Switch of Diodes," Trans. of IEICE, vol. E70, no. 8, pp. 744-754, 1987. 


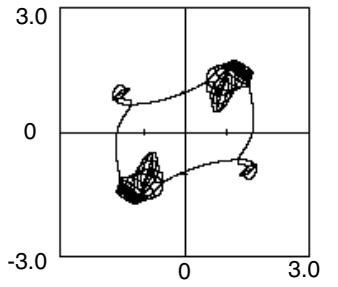

(1)

(2)
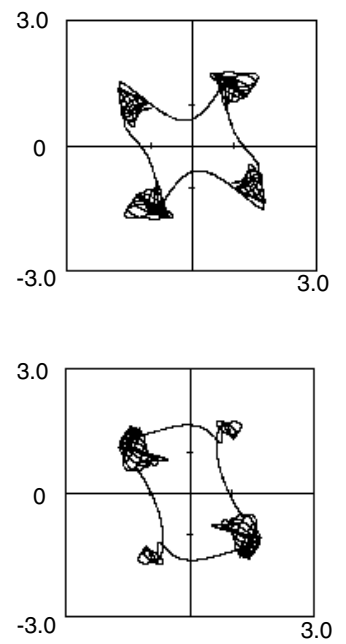

(3)

(a)
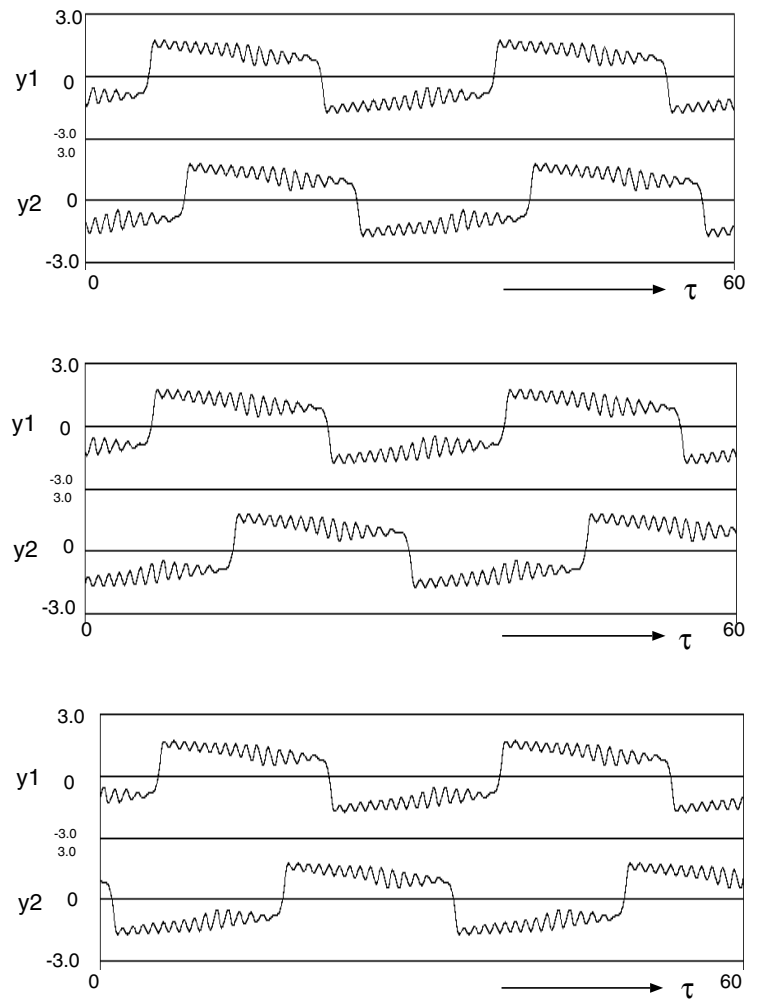

(b)

Fig. 9. Synchronization obtained from coupled Chua's circuits. (computer simulation results). $\alpha=15.6, \beta=50.0, \delta=0.0003, \delta=0.0014$, $m_{0}, m_{1}$. (1) First peak of $y_{2}$ is synchronized to the fourth bottom of $y_{1}$. (2) Eighth bottom of $y_{1}$. (3) Twelfth bottom of $y_{1}$. (a) Attractor on $y_{1}$ vs $y_{2}$ plane. (b) Timewaveform.

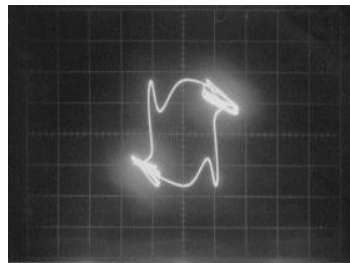

(a)

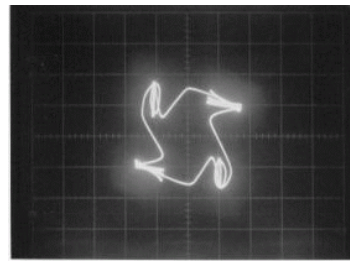

(a)

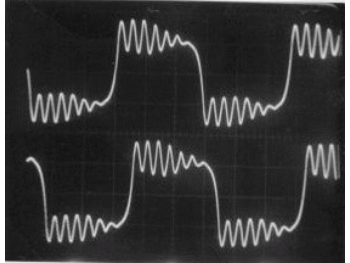

(b)

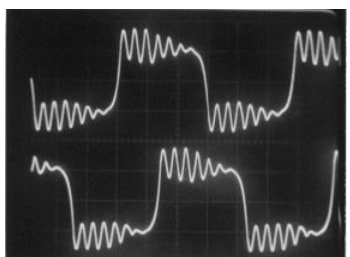

(b)
Fig. 6. Two examples of different synchronization states. (circuit experimental results). $L_{1}=10.56 \mathrm{mH}, L_{2}=1.28 \mathrm{H}, C_{1}=33.3 \mathrm{nF}$, $C_{2}=49.5 \mathrm{nF}$ and $g=515 \mathrm{mS}$. (a) Attractor on $v_{11}$ vs $v_{12}$ plane. Horizontal and vertical: $5 \mathrm{~V} /$ div. (b) Time waveform $v_{11}$ and $v_{21}$. Horizontal $0.5 \mathrm{~ms} / \mathrm{div}$ and vertical: $5 \mathrm{~V} / \mathrm{div}$.

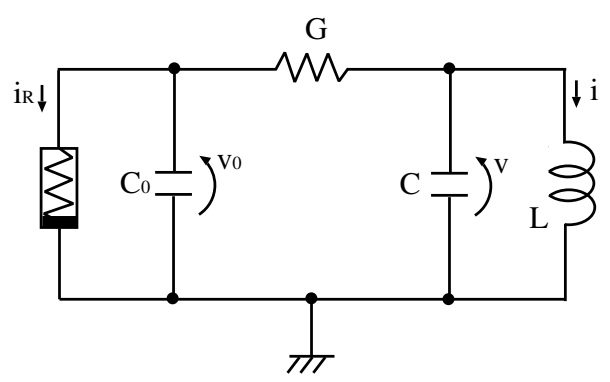

Fig. 7. Chua's circuit.

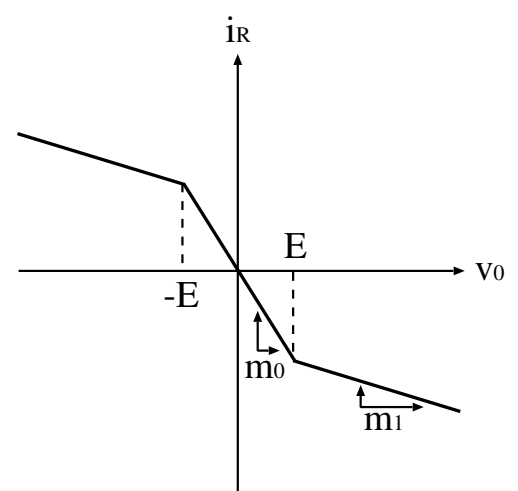

Fig. 8. $v-i$ characteristics of Chua diode. 